\title{
Four-dimensional cardiac imaging in living embryos via postacquisition synchronization of nongated slice sequences
}

\author{
Michael Liebling \\ California Institute of Technology \\ Beckman Institute \\ Biological Imaging Center \\ Mail Code 139-74 \\ Pasadena, California 91125
}

\author{
Arian S. Forouhar \\ Morteza Gharib \\ California Institute of Technology \\ Division of Engineering \& Applied Sciences \\ Options of Bioengineering and Aeronautics \\ Mail Code 138-78 \\ Pasadena, California 91125
}

\section{Scott E. Fraser}

Mary E. Dickinson

California Institute of Technology

Beckman Institute

Biological Imaging Center

Mail Code 139-74

Pasadena, California 91125

\begin{abstract}
Being able to acquire, visualize, and analyze 3D time series (4D data) from living embryos makes it possible to understand complex dynamic movements at early stages of embryonic development. Despite recent technological breakthroughs in 2D dynamic imaging, confocal microscopes remain quite slow at capturing optical sections at successive depths. However, when the studied motion is periodicsuch as for a beating heart-a way to circumvent this problem is to acquire, successively, sets of $2 \mathrm{D}+$ time slice sequences at increasing depths over at least one time period and later rearrange them to recover a 3D+time sequence. In other imaging modalities at macroscopic scales, external gating signals, e.g., an electro-cardiogram, have been used to achieve proper synchronization. Since gating signals are either unavailable or cumbersome to acquire in microscopic organisms, we have developed a procedure to reconstruct volumes based solely on the information contained in the image sequences. The central part of the algorithm is a least-squares minimization of an objective criterion that depends on the similarity between the data from neighboring depths. Owing to a wavelet-based multiresolution approach, our method is robust to common confocal microscopy artifacts. We validate the procedure on both simulated data and in vivo measurements from living zebrafish embryos. $\odot 2005$ Society of Photo-Optical Instrumentation Engineers. [DOl: 10.1117/1.2061567]
\end{abstract}

Keywords: confocal laser scanning microscopy; dynamic cardiac imaging; nongated synchronization; registration; wavelets.

Paper 05032R received Feb. 4, 2005; revised manuscript received Apr. 7, 2005; accepted for publication May 6, 2005; published online Oct. 4, 2005.

\section{Introduction}

Confocal laser scanning microscopy (CLSM) has emerged as a popular method for high-resolution imaging of fluorescent labels, particularly in thick or scattering samples. By placing a pinhole in the conjugate optical plane, before the detector, out-of-focus light from above and below the focal plane is rejected from the image, enhancing the axial resolution. ${ }^{1}$ By collecting images from defined optical slices at successive depths, the three-dimensional arrangement of fluorescentlylabeled structures can be derived. In traditional point scanning confocal systems, images are collected in a pixel-by-pixel manner and acquisition speeds for sequences with frame size $512 \times 512$ pixels are on the order of only a few frames per second. Recent advances in beam shaping, the availability of fast CCD line detectors, and the implementation of efficient hardware for data transmission have made possible the development of a fast laser scanning microscope, the LSM 5 LIVE. $^{2}$ A blade-shaped beam focused to a line (instead of a single point for conventional CLSM) permits the parallel ac-

Address all correspondence to Michael Liebling, California Institute of Technology, Biological Imaging Center, Beckman Institute, Mail Code 139-74, 118 S. Chester Ave., Pasadena, CA 91125. Tel.: (626) 395-2863, Fax: (626) 449-5163, E-mail: liebling@caltech.edu quisition of a whole line of pixels and reduces the scanning dimensionality to one direction. This microscope allows for the acquisition of $2 \mathrm{D}$ image sequences $(512 \times 512$ pixels $)$ at frame rates of up to 120 frames per second. This opens new avenues for a variety of fields.

In developmental biology, one major goal is to gain a better understanding of the mechanisms that influence the development of the cardiovascular system. In particular, it is desirable to assess the influence of genetic as well as epigenetic factors such as blood flow, heart wall forces, shear stress, etc. ${ }^{3,4}$ While the frame rates of typical confocal microscopes are suitable to study many dynamic processes occurring in living systems (e.g., cell migration, division, etc.), cell motions in the cardiovascular system (e.g., heart-wall motions, blood flow, etc.) typically occur at several millimeters per second, 2 to 3 orders of magnitude faster than cell migration. The significant improvement in frame rate offered by parallel scanning systems now makes it possible to collect image data from single optical sections of fast-moving structures. However, resolving rapid three-dimensional motions in real time still remains a challenge because it is not currently possible to scan the $z$ direction as fast as the $x y$ plane.

1083-3668/2005/10(5)/054001/10/\$22.00 @ 2005 SPIE 


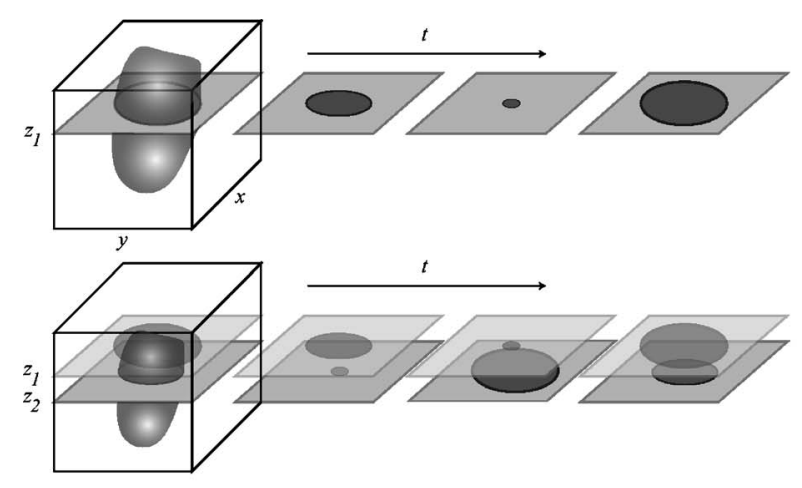

(a)

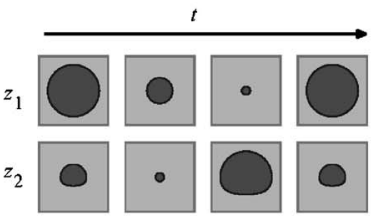

(b)
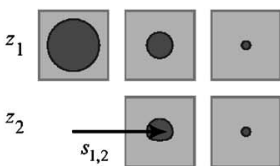

(c)

Fig. 1 (a) Nongated slice-sequence acquisition procedure. (b) Two 2D slice sequences before and (c) after synchronization.

Other imaging modalities such as magnetic resonance imaging (MRI), computerized tomography (CT), or ultrasound (US) suffer from similar limitations. However, if the imaged body undergoes the same deformation at regular intervals and the acquisition is always triggered at a particular phase in the cycle, it is possible to assemble the data to recover a whole volume over one full period. For larger organisms (from mice to humans), it is relatively easy to gate the acquisition with respect to electrocardiograms (ECG) or respiratory signals - a technique known as prospective gating or triggering-and reconstruct volumes at a fixed moment in the cycle. ${ }^{5-7}$ Remaining motion artifacts may then be reduced by the use of various elastic registration procedures that warp the spatial data. ${ }^{8-10} \mathrm{In}$ cases where gating is not possible or unreliable, nongated dynamic datasets have been registered by a variety of methods and for various purposes. For instance, in nuclear medicine, noise reduction may be performed through temporal averaging of nongated signals. ${ }^{11}$ Thompson and McVeigh have used the imaging data from flow-encoded MRI to retrospectively perform the gating. ${ }^{12}$ Using specific modifications to conventional MRI pulse sequences, it is also possible to generate and extract a signal that varies in synchrony with the cardiac cycle for later reconstruction. ${ }^{13-16}$ For CT, various methods have been developed, either to recover an imaged volume of the heart in a defined motion state at a single time point $^{17}$ or for $4 \mathrm{D}$ imaging, by tracking the projection's center of mass. ${ }^{18}$ ECG-free algorithms have also been used for US imaging. ${ }^{19,20}$

In this paper, we present a technique and the associated image processing that make it possible to reconstruct dynamic $3 \mathrm{D}$ volumes of microscopic objects that are periodically moving, using currently available CLSM technology. We sequentially acquire slice sequences at different depths and reassemble them a posteriori to recover dynamic 3D volumes. For smaller organisms, such as the zebrafish embryos we study, reliable triggering signals to gate the acquisition are difficult and cumbersome to acquire. We have therefore devised a method for postacquisition synchronization based upon information within the recorded nongated data itself.

Our synchronization algorithm registers pairs of slice sequences with respect to time by minimizing a least-squares intensity difference criterion. The core element of our method is thus reminiscent of standard methods for image registration, ${ }^{21}$ the latter being a particular instance of the more general problem of motion estimation; ${ }^{22-24}$ however, the nature of the data requires special adjustments in order to achieve stable and repeatable results with minimal operator input. First, since our problem naturally calls for periodic boundary conditions, we have to (automatically) crop the data to cover a whole number of periods. Second, the method must be robust to various acquisition artifacts that are specific to confocal microscopy. ${ }^{25-28}$ Last, the large amount of data that is involved imposes a subtle balance between memory and time constraints.

To solve some of the above requirements, our synchronization algorithms rely on the wavelet transform for robustness and rapid execution. For similar purposes, various authors have taken advantage of the favorable wavelet properties to implement affine and elastic registration algorithms of $2 \mathrm{D}$ or 3D datasets, ${ }^{29-36}$ although their methods are not directly related to ours.

The paper is organized as follows. In Sec. 2, we discuss the measurement process as well as the requirements and limitations of the method. In Sec. 3, we present the synchronization algorithm, along with the tools that are required for period determination and noise reduction. In Sec. 4, we present two experiments, one based on a simulated acquisition procedure and the second, based on experimental in vivo measurements. Finally, we discuss the method's overall performance and limitations as well as further developments.

\section{Measurement}

\subsection{Measurement Strategy}

We image a slice of a $3 \mathrm{D}$ object, subject to periodic motions and deformations over typically two to four periods. We assume that the object is given by the local intensity $I(\mathbf{x}, z, t)$ $\in\left[0, I_{\max }\right]$, with $\mathbf{x}=(x, y)$ and that the periodic deformations are such that at any fixed spatial position $(\mathbf{x}, z)$ we have

$$
|I(\mathbf{x}, z, t)-I(\mathbf{x}, z, t+T)| \ll I_{\max },
$$

where $T$ is the deformation period. Between acquisitions, the object is moved axially ( $z$ direction) to be sequentially imaged in its entirety (see Fig. 1). Because the acquisition is triggered at a random moment in the heart cycle, the different sequences are not synchronized. The measured intensity can be modeled by 


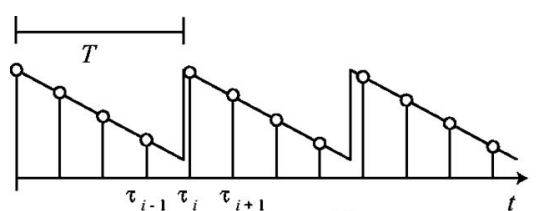

(a)
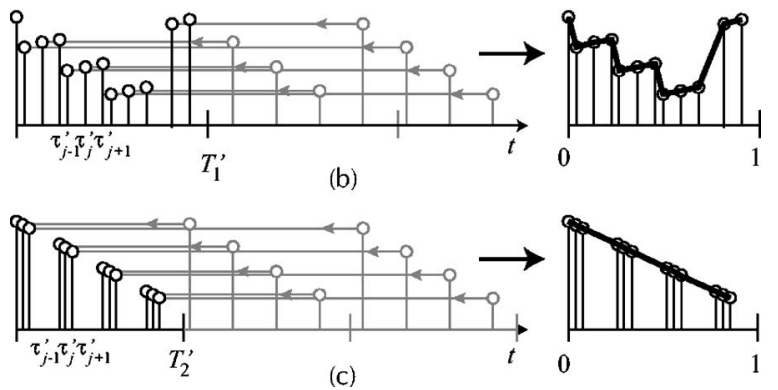

Fig. 2 Dispersion measure for period estimation. (a) Sampled intensity variation over time (with unknown period $T$ ) at one location $\mathbf{x}$. (b) For a candidate period $T_{1}^{\prime}$ the samples are brought back to the first period (phase locking). The dispersion of the samples is given by the length of the curve that joins the newly ordered samples (bold curve). (c) When the candidate period corresponds to the actual period, i.e., $T_{2}^{\prime}=T$, the dispersion of the samples, hence the length of this curve, is minimized.

$$
I_{m}\left(\mathbf{x}, z_{k}, t\right)=\iiint I\left(\mathbf{x}^{\prime}, z, t-s_{k}\right) \operatorname{PSF}\left(\mathbf{x}-\mathbf{x}^{\prime}, z_{k}-z\right) \mathrm{d} \mathbf{x}^{\prime} \mathrm{d} z
$$

where the $s_{k}$ are the unknown time shifts (defined modulo the period) at each measured depth $z_{k}=k h, k=0, \ldots, N_{z}-1$, with $h$ the axial slice spacing. The ideal point spread function (PSF) can be expressed by the Dirac delta function

$$
\operatorname{PSF}(\mathbf{x}, z)=\delta(\mathbf{x}) \delta(z) .
$$

In practice, the sampling is not ideal and we assume that the PSF has a spatial extent in the $z$ direction that is larger than the axial slice spacing $h$.

The subsequent algorithms aim at finding the unknown sequence $s_{k}$ in order to retrieve the original volume $I(\mathbf{x}, z, t)$ from the measurements $I_{m}\left(\mathbf{x}, z_{k}, t\right)$.

\subsection{Synchronization}

The core of the synchronization procedure rests on the registration of slice-sequence pairs with respect to time. We seek solutions that, for a given time shift, maximize the similarity (in some metric to be defined) between two adjacent slices. This similarity hypothesis is reasonable if the axial sampling step $h$ (the distance between two adjacent slices) is smaller than the PSF extent in $z$ or that the object undergoes sufficiently smooth and homogeneous deformations. Indeed, while the axial resolution drops as the axial extent of the PSF increases, the similarity between two adjacent slices improves as both measurements contain information from the same physical region. For the same slice spacing, ideal sampling induces better axial resolution, to the detriment of the similarity hypothesis. While a rigorous investigation about all possible motions that may or may not be imaged using this tech-

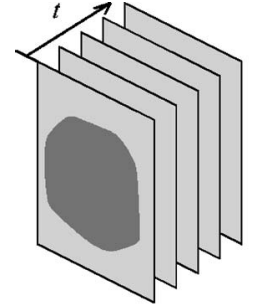

(a)

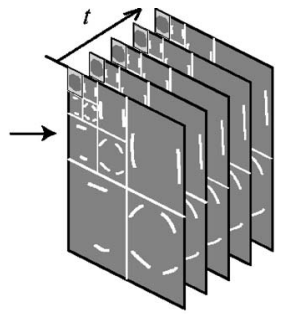

(b)

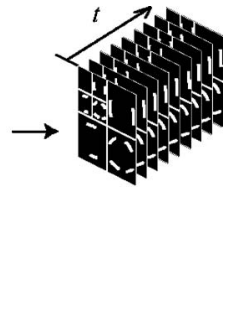

(c)
Fig. 3 Synchronization based on wavelet coefficients. (a) Original slice sequence at a given depth. (b) A 2D wavelet transform is applied to each frame. (c) Fine-scale wavelet coefficients are discarded (data reduction) as well as the low-pass coefficients at the coarsest scale. A threshold is applied to the remaining coefficients to increase robustness to noise. The reduced data is interpolated to increase synchronization accuracy.

nique is beyond the scope of this paper, we have heuristically determined that a unique and correct dynamic object can be recovered in the case of periodic, continuous, and homogeneous transforms even in the unfavorable case of ideal sampling. In Sec. 4.1, we present a simulation that supports this observation. Deformations that are nonhomogeneous with respect to the $z$ axis may result in incorrect reconstructions when the axial slice spacing $h$ is too large, that is, larger than the axial extent of the PSF. In practice, such cases may only be dealt with by considering a region of interest where the deformation is known to be homogeneous (see Sec. 4.1) or by the use of external information (ECG, etc.).

\section{Algorithms}

In this section we present the synchronization methodology. The following steps are involved in the data processing:

1. period determination, data interpolation, and cropping,

2. determination of relative shifts between pairs of slices,

3. determination of the slices' absolute shifts with respect to the first slice,

4. synchronization and postprocessing.

\subsection{Period Determination}

In order to ensure proper synchronization, the heartbeat period must be known precisely and be the same for all slice sequences at different depths. The image sequences are acquired at times $\tau_{i}=i h_{\tau}, i=0, \ldots, N_{\tau}-1$, where $h_{\tau}$ is the acquisition sampling step and $N_{\tau}$ is the number of acquired frames. We achieved precise and automatic period determination by use of a technique inspired from astronomy. ${ }^{37,38}$ For a given slice sequence and a candidate period $T^{\prime}$, the time positions of every pixel are brought back to the first period (phase locking)

$$
\tau_{i}^{\prime}=\tau_{i}-\left\lfloor\tau_{i} / T^{\prime}\right\rfloor T^{\prime}
$$

and a bijective mapping $i=i(j)$ [respectively $j=j(i)$ ] is defined such that $\tau_{j-1}^{\prime} \leqslant \tau_{j}^{\prime} \leqslant \tau_{j+1}^{\prime}$. An estimate of the phaselocked signal's dispersion is given by the length of the graph $\left(\tau_{j}^{\prime}, I_{m}\left[\mathbf{x}, z_{k}, \tau_{i(j)}\right]\right)_{j=0, \ldots, N_{\tau}-1}$ that joins the newly ordered samples on a normalized time scale (see Fig. 2), cumulated over the whole image, i.e., 


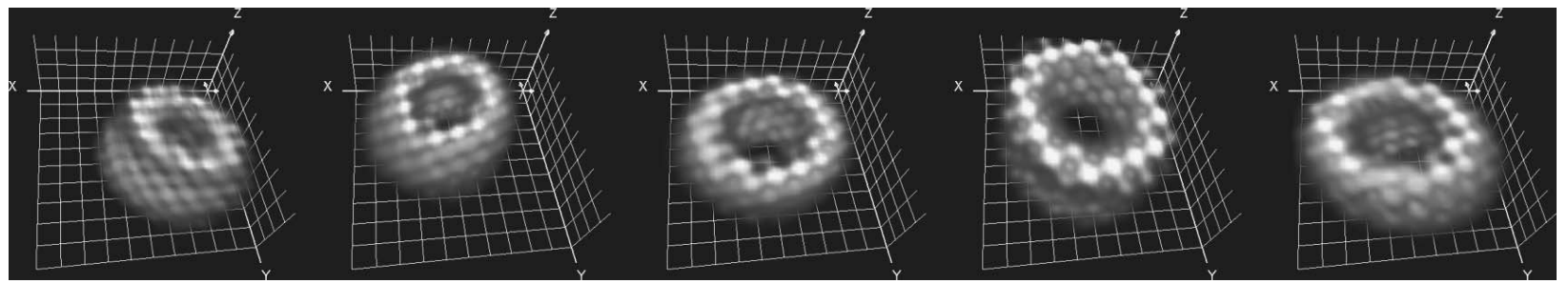

Fig. 4 Five time points of a simulated heart-tube deformation cycle (homogeneous transform). The corresponding movie is available at Ref. 62.

$$
\begin{aligned}
D\left(z_{k}, T^{\prime}\right)= & \sum_{\mathbf{m} \in \mathbf{Z}^{2}} \sum_{j=1}^{N_{\tau^{-}}}\left\{\left|I_{m}\left[\mathbf{x}_{\mathbf{m}}, z_{k}, \tau_{i(j)}\right]-I_{m}\left[\mathbf{x}_{\mathbf{m}}, z_{k}, \tau_{i(j-1)}\right]\right|^{2}\right. \\
& \left.+\left|\tau_{j}^{\prime}-\tau_{j-1}^{\prime}\right|^{2} / T^{\prime 2}\right\}^{1 / 2},
\end{aligned}
$$

with $\mathbf{x}_{\mathbf{m}}=\mathbf{m} h_{x y}$. Here, for simplicity, we consider that the sampling step in the $0 x y$ plane, $h_{x y}$, is 1 . The correct period $T\left(z_{k}\right)$ is found by minimizing the above expression, viz.,

$$
T\left(z_{k}\right)=\arg \min _{T^{\prime}} D\left(z_{k}, T^{\prime}\right)
$$

Starting from an initial guess of the range $T\left(z_{k}\right)$ $\in\left[T_{\min }, T_{\text {max }}\right]$, we solve Eq. (6) iteratively using a combined parabolic and golden section search algorithm, ${ }^{39-41}$ which usually converges to a subsampling-step accuracy in less than 10 iterations.

In order for the periodic boundary conditions in the time direction to be applied during subsequent operations, we crop and resample the data to cover an integer number of periods. We used linear spline interpolation, which offers a fair compromise between the accuracy of higher order interpolation schemes and the time efficiency of nearest neighbor interpolation. $^{42,43}$ The samples are taken at times $t_{i}=i h_{t}, i$ $=0, \ldots, N_{t}-1$, with sampling step $h_{t}=L / N_{t}$, where $N_{t}$ is the number of considered frames over the total time $L=N_{T} T$ and $N_{T}$ the number of considered periods. This also allows for temporal stretching or compression in cases where the periods at different depths are not the same. From this point onward, we consider that the measured signal $I_{m}\left(\mathbf{x}, z_{k}, t\right)$ is known for $\mathbf{x} \in \mathbf{R}^{2}$ and $t \in[0, L)$ (possibly via the interpolation of samples that are uniformly distributed over that domain) and that periodic boundary conditions in time apply.

\subsection{Determination of Relative Shifts}

Our automatic synchronization algorithm is based on the minimization of an objective criterion to measure the similarity between the data from neighboring depths $z_{k}$ and $z_{k^{\prime}}$. We have chosen a least-squares criterion that has been shown to be effective for registration algorithms ${ }^{44}$

$$
Q_{k, k^{\prime}}(s)=\iint_{\mathbf{R}^{2}} \int_{0}^{L}\left|I_{m}\left(\mathbf{x}, z_{k}, t\right)-I_{m}\left(\mathbf{x}, z_{k^{\prime}}, t-s\right)\right|^{2} \mathrm{~d} t \mathrm{~d} \mathbf{x}
$$

where the shift $s \in \mathbf{R}$. We can further write

$$
\begin{aligned}
Q_{k, k^{\prime}}(s)= & \iint_{\mathbf{R}^{2}} \int_{0}^{L}\left|I_{m}\left(\mathbf{x}, z_{k}, t\right)\right|^{2}+\left|I_{m}\left(\mathbf{x}, z_{k^{\prime}}, t-s\right)\right|^{2} \mathrm{~d} t \mathrm{~d} \mathbf{x} \\
& -2 \iint_{\mathbf{R}^{2}} \int_{0}^{L} I_{m}\left(\mathbf{x}, z_{k}, t\right) I_{m}\left(\mathbf{x}, z_{k^{\prime}}, t-s\right) \mathrm{d} t \mathrm{~d} \mathbf{x} \\
= & C-2 \iint_{\mathbf{R}^{2}} \int_{0}^{L} I_{m}\left(\mathbf{x}, z_{k}, t\right) I_{m}\left(\mathbf{x}, z_{k^{\prime}}, t-s\right) \mathrm{d} t \mathrm{~d} \mathbf{x},
\end{aligned}
$$

where the integral of the second quadratic term does not depend on $s$ because of the periodicity with respect to time. Since the above expression has the form of a correlation and periodic boundary conditions apply, we can compute $Q_{k, k^{\prime}}(s)$ (up to the constant $C$ ) for a number of regularly spaced shifts $s=i h_{t}$, with $h_{t}=L / N_{t}, i=0, \ldots, N_{t}-1$, at a limited cost using the fast Fourier transform (FFT). The relative shifts $s_{k, k^{\prime}}$ between any two pairs of $z$ slices are obtained by finding the shifts $s$ that minimizes $Q_{k, k^{\prime}}(s)$. They may be represented by the antisymmetric matrix $\mathbf{S}$, whose elements are

$$
s_{k, k^{\prime}}=\arg \min _{s=k T, k=1, \ldots, N_{t}} Q_{k, k^{\prime}}(s) .
$$

Note that this matrix not only includes slice-sequence pairs that are immediate neighbors but also pairs that lie farther apart. We also compute the correlation for such slices in order to reduce synchronization errors that may quickly propagate due to the sequential alignment and the limited discrete steps the FFT imposes.

Before we derive the method for the determination of the shifts relative to the first slice sequence (absolute shifts), we refine the above correlation technique to make it time and memory effective as well as robust. Indeed, computing Eqs. (8) and (5) naively would require considerable time and memory resources as the multidimensional data rapidly exceeds the storage capacity of even the latest available desktop computers. Another concern that complicates the equations' direct implementation is that the images are corrupted by noise. As a consequence, the objective functions are as well. Yet another caveat is the presence of features that are characteristic of the studied structure but do not comply with the similarity hypothesis. For example, red blood cells are confined to the inside of the heart tube and have a movement that is in synchrony with the heart movement, however, the individual cells do not occupy the same positions from slice to slice. The correct extremum determination is thus severely affected. We have chosen to take advantage of the 


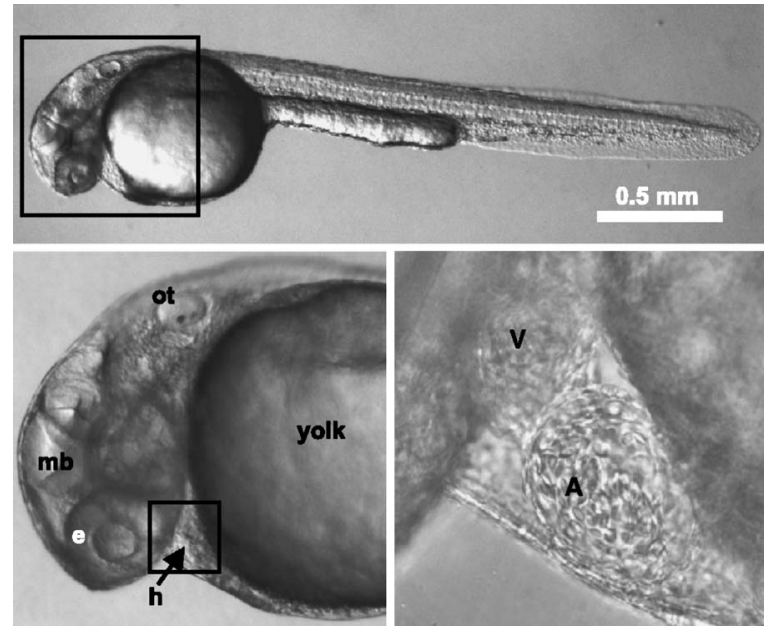

Fig. 5 Bright-field images of a zebrafish embryo at 48 h.p.f.; $h$ =heart; $\mathrm{e}=$ eye; $\mathrm{mb}=$ midbrain; $\mathrm{ot}=$ otocyst; yolk=yolk mass; $\mathrm{A}$ = atrium; $\mathrm{V}=$ ventricle.

multiresolution $^{45}$ and noise decorrelation ${ }^{46,47}$ properties that the wavelet decomposition offers to solve these issues.

We consider a separable orthogonal wavelet basis of $L_{2}\left(\mathbf{R}^{2}\right)$

$$
\left\{\psi_{j, \mathbf{m}}^{1}(\mathbf{x}), \psi_{j, \mathbf{m}}^{2}(\mathbf{x}), \psi_{j, \mathbf{m}}^{3}(\mathbf{x})\right\}_{j \in \mathbf{Z}, \mathbf{m} \in \mathbf{Z}^{2}}
$$

where the two-dimensional wavelets

$$
\psi_{j, \mathbf{m}}^{p}(\mathbf{x})=\frac{1}{2^{j}} \psi^{p}\left(\frac{\mathbf{x}}{2^{j}}-\mathbf{m}\right)
$$

are constructed with separable products of the 1D scaling function $\phi(x)$ and wavelet $\psi(x)$

$$
\psi^{1}(\mathbf{x})=\phi(x) \psi(y), \psi^{2}(\mathbf{x})=\psi(x) \phi(y), \psi^{3}(\mathbf{x})=\psi(x) \psi(y) .
$$

For the sake of brevity, we index the basis functions with a single index $\mathbf{k}$ that includes the scale $j \in \mathbf{Z}$, translation $\mathbf{m}$ $\in \mathbf{Z}^{2}$, and wavelet type $p \in\{1,2,3\}$ :

$$
\psi_{\mathbf{k}}(\mathbf{x})=\psi_{j, \mathbf{m}}^{p}(\mathbf{x}), \quad \mathbf{k}=(p, j, \mathbf{m}) .
$$

With this notation, we may expand an image at a fixed depth $z_{k}$ and time point $t$ in the wavelet basis as

$$
I_{m}\left(\mathbf{x}, z_{k}, t\right)=\sum_{\mathbf{k}} c_{k, \mathbf{k}}(t) \psi_{\mathbf{k}}(\mathbf{x})
$$

where the coefficients are given by the inner products (wavelet transform)

$$
\begin{aligned}
c_{k, \mathbf{k}}(t) & =\left\langle I_{m}\left(\cdot, z_{k}, t\right), \psi_{\mathbf{k}}\right\rangle \\
& =\iint I_{m}\left(\mathbf{x}, z_{k}, t\right) \psi_{\mathbf{k}}(\mathbf{x}) \mathrm{d} \mathbf{x} .
\end{aligned}
$$

Since the basis functions are orthogonal, i.e., $\left\langle\psi_{\mathbf{k}}, \psi_{\mathbf{k}^{\prime}}\right\rangle$ $=\delta_{\mathbf{k}, \mathbf{k}^{\prime}}$, we may rewrite Eq. (8) as

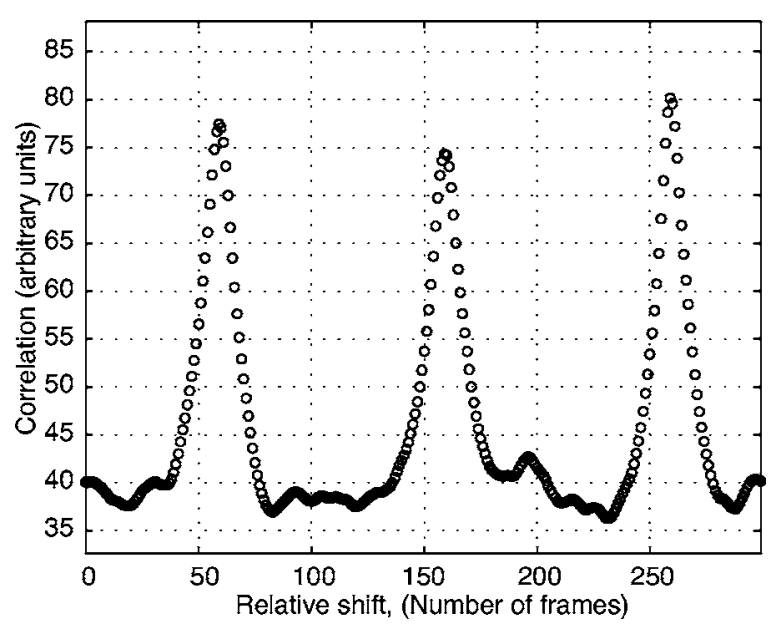

Fig. 6 Experimental correlation curve [see Eq. (8)] for two adjacent slice sequences. The maximum correlation occurs for a shift of 259 frames.

$$
\begin{aligned}
Q_{k, k^{\prime}}\left(j h_{t}\right) & =C-2 \int_{0}^{L} \sum_{\mathbf{k}} c_{k, \mathbf{k}}(t) c_{k^{\prime}, \mathbf{k}}\left(t-j h_{t}\right) \mathrm{d} t \\
& \approx C-2 h_{t} \sum_{\mathbf{k}} \sum_{i=0}^{N_{t}-1} c_{k, \mathbf{k}}\left(i h_{t}\right) c_{k^{\prime}, \mathbf{k}}\left[(i-j) h_{t}\right] .
\end{aligned}
$$

In practice, we only consider a finite number of scales and translations for $\mathbf{k}$ (because of the finite resolution and support of the image, and appropriate boundary conditions). Furthermore, we discard the fine resolution coefficients thus downsizing the data's complexity to a tractable size (see Fig. 3). Since wavelet transforms induce concise signal representations we make sure that the most important information is still present. Also, at coarse scales, individual blood cells are not resolved. Since they are confined to the inside of the heart tube, their global position contributes to a useful correlation signal. However, since confocal images are subject to bleaching (whose consequence is the presence of a nonuniform background), we discard the low-pass coefficients (that contain most of the background energy) as well. We then apply a soft threshold to the remaining coefficients to limit the influence of other noise sources.

Similarly, we may apply Eq. (5) to the reduced data set of wavelet coefficients instead of the sampled image pixels, i.e.,

$$
\begin{aligned}
\widetilde{D}\left(z_{k}, T^{\prime}\right)= & \sum_{\mathbf{k}} \sum_{j=1}^{N_{\tau}-1}\left\{\left|c_{k, \mathbf{k}}\left[\tau_{i(j)}\right]-c_{k, \mathbf{k}}\left[\tau_{i(j-1)}\right]\right|^{2}\right. \\
& \left.+\left|\tau_{j}^{\prime}-\tau_{j-1}^{\prime}\right|^{2} / T^{\prime 2}\right\}^{1 / 2},
\end{aligned}
$$

thus gaining robustness, reducing the required memory, and decreasing the computation time. Although Eqs. (5) and (18) are not formally equivalent, the latter may be compared to applying the former to a sequence of images whose main features (edges) have been enhanced. Indeed, the wavelet transform essentially acts as an oriented differential operator at multiple scales.

We did not notice significant differences in the overall behavior of the algorithm depending on the choice of the wave- 
let basis, which must, however, be orthogonal to ensure validity of Eq. (17). We chose to work with the Daubechies 9/7 wavelets. $^{48}$ Although they are not orthogonal (but nearly ${ }^{49}$ ), they have good approximation properties and are symmetric. The latter property allows the implementation of an algorithm which does not require that the image size be a multiple of a power of two ${ }^{50}$ and which is thus well suited for region-ofinterest processing.

Finally, to increase the synchronization accuracy, we linearly interpolate the processed wavelet coefficients with respect to time in order to obtain a finer time step when computing Eq. (17). This interpolation is fast since the amount of data is reduced.

\subsection{Absolute Shifts Determination}

To determine the slice-sequence shifts with respect to the uppermost sequence (absolute shifts) $s_{k}$, we consider their relation to the relative shifts $s_{k, k^{\prime}}$ :

$$
s_{1}=0
$$

$$
s_{k}-s_{k^{\prime}}=s_{k, k^{\prime}}, \quad \text { with } k, k^{\prime}=1, \ldots, N_{z} \text { and } k<k^{\prime} .
$$

Since slice-sequence pairs that are separated by a larger depth are less trustworthy, we assign different weights $w_{\left|k^{\prime}-k\right|}$ to equations that involve the estimated shifts $s_{k, k^{\prime}}$ depending on the distance $j=\left|k^{\prime}-k\right|$. We set lower weights $w_{j}$ to equations for slice pairs less likely to exhibit similarities, that is, when the distance $\left|k^{\prime}-k\right|$ between them increases. For a system with $N_{z}=5$ and $w_{j}=0$ for $j>2$, we can rewrite Eq. (19) in matrix form

$$
\underbrace{\left[\begin{array}{ccccc}
1 & 0 & 0 & 0 & 0 \\
1 & -1 & 0 & 0 & 0 \\
0 & 1 & -1 & 0 & 0 \\
0 & 0 & 1 & -1 & 0 \\
0 & 0 & 0 & 1 & -1 \\
1 & 0 & -1 & 0 & 0 \\
0 & 1 & 0 & -1 & 0 \\
0 & 0 & 1 & 0 & -1
\end{array}\right]}_{\mathbf{A}} \underbrace{\left(\begin{array}{c}
s_{1} \\
s_{2} \\
s_{3} \\
s_{4} \\
s_{5}
\end{array}\right)}_{\mathbf{t}}=\underbrace{\left[\begin{array}{c}
0 \\
s_{1,2} \\
s_{2,3} \\
s_{4,5} \\
s_{1,3} \\
s_{2,4} \\
s_{3,5}
\end{array}\right]}_{\mathbf{s}}
$$

along with the diagonal weighting matrix

$$
\mathbf{W}=\operatorname{diag}\left(1, w_{1}, w_{1}, w_{1}, w_{1}, w_{2}, w_{2}, w_{2}\right) .
$$

We determine the weighted least-squares solution of Eq. (20), which is equivalent to solving

$$
\mathbf{A}^{\dagger} \mathbf{W}^{\dagger} \mathbf{W A t}=\mathbf{A}^{\dagger} \mathbf{W}^{\dagger} \mathbf{W} \mathbf{s}
$$

where $(\cdot)^{\dagger}$ denotes transposition. Equation (20) may easily be modified to include supplementary information (not imageintrinsic) that may become available in the future, such as ECGs. Depending on the accuracy of the signals, we may then set appropriate weights in Eq. (21).

\subsection{Synchronization and Postprocessing}

The original slice sequences are finally circularly shifted by the computed absolute shifts (using linear interpolation and resampling). The synchronized data may then be visualized using 4D-capable software packages. ${ }^{51}$ Noise reduction steps may be applied. We made use of a rolling-ball background removal algorithm (see Refs. 52 and 53) to normalize the background. The 4D data series may also be analyzed to follow individual cell movements. The higher dimensionality of the data should also make it possible to take advantage of more sophisticated noise removal algorithms that have proven to be effective for other high-dimensional modalities. ${ }^{54,55} \mathrm{Fi}$ nally, the synchronized data might be suitable for subsequent deconvolution.

\section{Results and Discussion}

\subsection{Simulation}

We validate our approach by simulating the acquisition procedure on a periodically deformed test body. We have considered the following, much simplified, heart-tube phantom. At time $t=0$, the contributing intensity at every location $(\mathbf{x}, z)$ is given by

$$
\begin{aligned}
I(\mathbf{x}, z, 0)= & I_{0} \beta^{3}\left(\frac{\left(x^{2}+y^{2}\right)-r_{0}(z)}{w / 4}\right) \\
& \times\left[1+\gamma \cos \left(2 \pi f_{d} x\right) \cos \left(2 \pi f_{d} y\right) \cos \left(2 \pi f_{d} z\right)\right]
\end{aligned}
$$

where the central wall radius is given by

$$
r_{0}(t, z)=R_{0}+\Delta_{R} \sin (2 \pi \alpha z)
$$

and where $w$ is the wall thickness, $\alpha$ controls the tube's geometry, $R_{0}$ is the average tube radius, $\Delta_{R}$ is the radius movement amplitude, $\gamma$ is the amplitude of a regular pattern of frequency $f_{d}$, and the cubic B-spline ${ }^{56}$ is given by

$$
\beta^{3}(x)= \begin{cases}2 / 3-|x|^{2}+|x|^{3} / 2, & 0 \leqslant|x|<1 \\ (2-|x|)^{3} / 6, & 1 \leqslant|x|<2 \\ 0, & 2 \leqslant|x| .\end{cases}
$$

Typical heart motions include rotation, expansion, contraction, and shear. ${ }^{57}$ We model the intensity at subsequent times by a general periodic affine transformation of the coordinate system corresponding to an homogeneous deformation of the original body. The intensity at position $(x, y, z)$ and time $t$ is given by

$$
I(\mathbf{x}, z, t)=I\left(\mathbf{x}^{\prime}, z^{\prime}, 0\right)
$$

where, using homogeneous coordinates, ${ }^{58}$ 

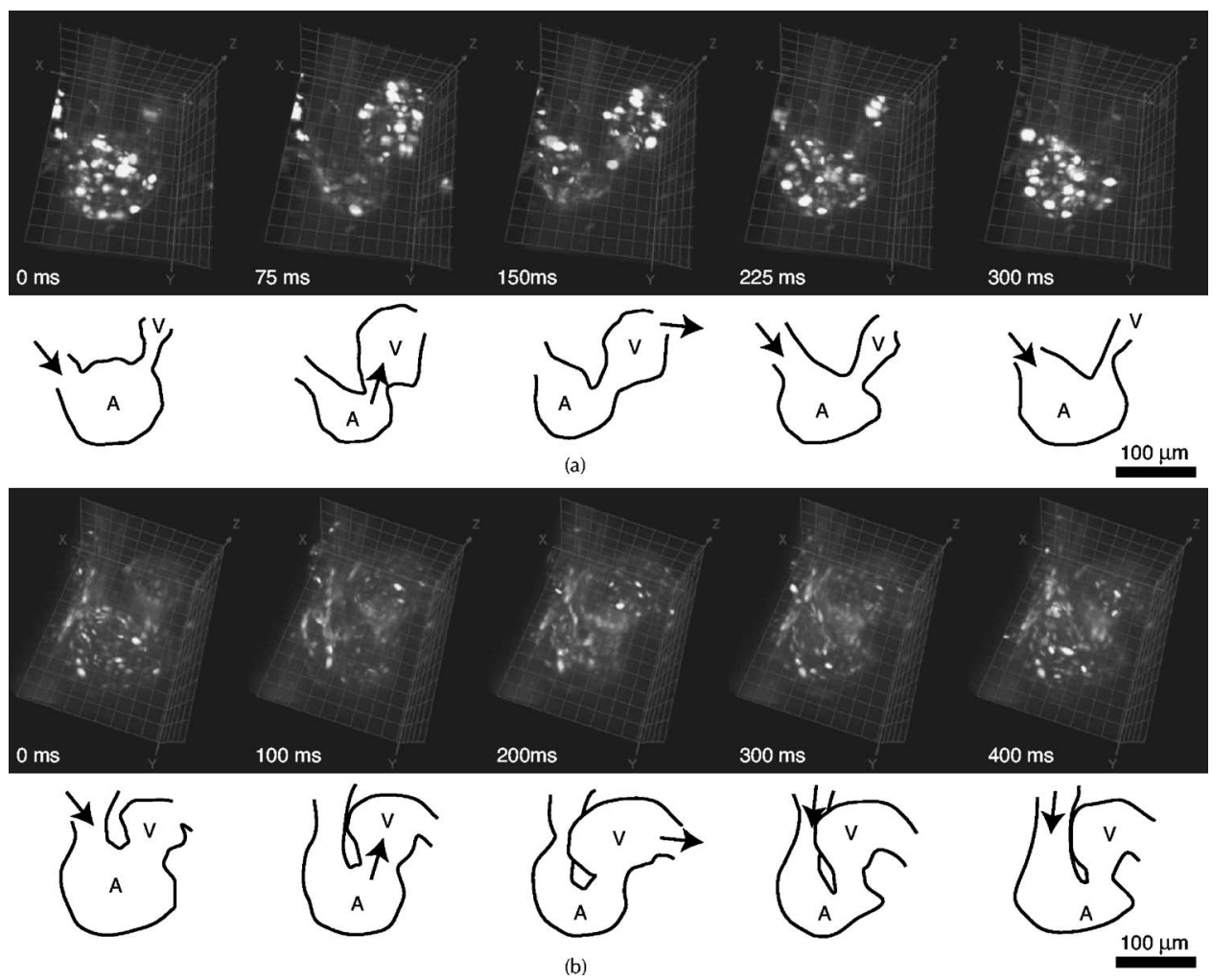

Fig. 7 Reconstructed 4D datasets of beating 48 h.p.f. $\operatorname{Tg}($ gata1::GFP) zebrafish hearts. In the first frame, the red blood cells fill the atrium. In the following frames, the red blood cells are pumped through the atrial ventricular canal into the ventricle. The atrium fills again while the cycle is completed. The grid spacing is $20 \mu \mathrm{m}$. Movies are available at Ref. 62.

$$
\left(\begin{array}{c}
x^{\prime} \\
y^{\prime} \\
z^{\prime} \\
1
\end{array}\right)=\left(\begin{array}{cccc}
a_{11}(t) & a_{12}(t) & a_{13}(t) & a_{14}(t) \\
a_{21}(t) & a_{22}(t) & a_{23}(t) & a_{24}(t) \\
a_{31}(t) & a_{32}(t) & a_{33}(t) & a_{34}(t) \\
0 & 0 & 0 & 1
\end{array}\right) \cdot\left(\begin{array}{c}
x \\
y \\
z \\
1
\end{array}\right)
$$

The time-periodic affine transformation matrix $\mathbf{A}(t)$ can be decomposed as a combination of translation, rotation, scaling, and shear:

$$
\mathbf{A}(t)=\mathbf{T}(t) \cdot \mathbf{R}(t) \cdot \mathbf{H}(t) \cdot \mathbf{C}(t)
$$

where $\mathbf{T}(t), \mathbf{R}(t), \mathbf{H}(t)$, and $\mathbf{C}(t)$ are the matrices corresponding to the respective transformations. Twelve parameters control the deformation matrix and each of them is a periodic function of time, which we specify through the coefficients of its Fourier series (see Appendix A). The latter may be chosen randomly to cover the full range of possible transformations. In Fig. 4, we show several time points of such a random, periodic, and continuous deformation cycle that includes shear, rotation, translation, and scaling.

To assess the performance of our method, we generated a set of 100 deformation cycles using at each time different (normally distributed) random variables for the second and third harmonics of each parameter function, as well as random shifts $\bar{s}_{k} \sim \operatorname{Un}(-T, T)$ (uniform probability distribution). We considered the simplified PSF of Eq. (3) with $N_{z}=20, N_{t}$ $=40, h_{r}=1$ (normalized time units), and a period $T=19.5$. From these simulated measurements, we then applied our algorithm (using 80 time points to compute the correlations, that is, after the cropping step, approximately $2 \times$ oversampling) to retrieve the shifts $s_{k}$. Since the true absolute shifts were known, we could compute the absolute error using the following formula (which takes into account periodicity, e.g., comparing shifts $s_{1}=\delta$ and $s_{2}=T-\delta$ yields an error $\epsilon=2 \delta$ ):

$$
\epsilon=\min \left(\left|\mathcal{W}_{T}\left(\bar{s}_{k}\right)-\mathcal{W}_{T}\left(s_{k}\right)\right|, T-\left|\mathcal{W}_{T}\left(\bar{s}_{k}\right)-\mathcal{W}_{T}\left(s_{k}\right)\right|\right)
$$

where $\mathcal{W}_{T}(x)=x-\lfloor x / T\rfloor T$. The mean error over the 100 experiments was $\bar{\epsilon}=0.31 \pm 0.08$ frames. This result confirms that for the vast class of periodic homogeneous transforms our method is highly reliable, even when the considered sampling is ideal, i.e., when there is no axial overlap of the PSF. The error may be reduced by linearly interpolating the wavelet coefficients at a finer sampling rate in time. For different oversampling rates, we obtained the following errors: $\bar{\epsilon}_{1 \times}$ $=0.41 \pm 0.12, \bar{\epsilon}_{2 \times}=\bar{\epsilon}=0.31 \pm 0.08, \bar{\epsilon}_{4 \times}=0.27 \pm 0.06$, and $\bar{\epsilon}_{8 \times}$ $=0.25 \pm 0.06$. However, visual inspection of the reconstructions from in vivo measurements showed no significant improvement of the results above $2 \times$ oversampling. Also, although the accuracy of correlation-based registration methods is known to be inherently limited, ${ }^{59}$ in practice, the current limiting factors are the irregularities in the heartbeat periodicity of the biological samples themselves.

\subsection{Experimental Measurements}

With the aim of a better understanding of the zebrafish cardiac development, we applied our method to slice sequences from an early embryonic, 48 hours post fertilization (h.p.f.), beat- 
ing heart. In Fig. 5, we show a bright-field microscopy image of a 48 h.p.f. zebrafish embryo where the heart position has been indicated. The study focuses on zebrafish for several reasons: they are vertebrates that reproduce externally and rapidly, they are relatively transparent, and it is possible to genetically engineer fish strains that express vital fluorescent markers in specific tissues (for instance, heart wall, or blood cells). Here, we have chosen to study $\operatorname{Tg}($ gata $1:$ GFP) zebrafish embryos whose endo- and myocardial cells as well as erythrocytes are fluorescent. ${ }^{60}$ The embryos were anesthetized in order to limit the imaged movements to those of the heart. Images were acquired using a Zeiss LSM 5 LIVE laser scanning microscope prototype ${ }^{2}$ at a frame rate of $151 \mathrm{~Hz}$ for the duration of three to four heartbeats. The images had 256 $\times 256$ pixels and a sampling step of $0.9 \mu \mathrm{m}$ per pixel $(40$ $\times$ AchroPlan water-immersion lens $\mathrm{NA}=0.8$ ). The stage was then moved axially in increments of $5 \mu \mathrm{m}$ before a new sequence was acquired. A total of about 20 positions could be imaged per embryo. A complete description of the experimental aspects of the measurement procedure is reported elsewhere. $^{61}$

The heartbeat of the studied zebrafish appeared to remain steady over the usual acquisition time for one slice (three to four heartbeats). However, we observed changes in the rate of up to about $10 \%$ between the sequence at the first and last $z$ position. Once identified, these variations - mainly due to ambient temperature changes - could subsequently be controlled to limit the period change. We considered three periods per slice sequence. In Fig. 6, we show the experimental correlation curve for one slice-sequence pair. The curve's three main maxima correspond to admissible periodic shifts (one peak shift per imaged heartbeat).

In Fig. 7, we show the rendering over five frames of two reconstructed embryo hearts. Mostly erythrocytes, but also endo- and myocardial cells are fluorescent and visible. The orientation with respect to the $z$ axis is different for the two samples, yet the reconstructions show similar features, which supports the hypothesis that the method is suitable for accurate imaging of the wall deformations. These reconstructed images allow the visualization of complex flow and wall movement patterns that previously could not be studied.

The computation time on a $2-\mathrm{GHz}$ PowerPC G5, for a set of 20 slice sequences of size $256 \times 256$ pixels and 220 time frames, is distributed as follows: preprocessing (wavelet transform): $1 \mathrm{~min}$; period retrieval: $10 \mathrm{~s}$; time interpolation, resampling of wavelet coefficients, and FFT: 7 s; shift determination: $7 \mathrm{~s}$ (absolute shift determination takes less than $0.01 \mathrm{~s}$ ); original data shifting, interpolation, and sampling: $40 \mathrm{~s}$. Finally, our implementation's memory requirements (RAM) are below $512 \mathrm{MB}$ for the above dataset.

\section{Conclusion}

We have presented a procedure for the synchronization of nongated confocal slice sequences to build dynamic 3D volumes. We have investigated the ability of our method to achieve this goal and found that it performs well. We have validated the approach both through simulation and in vivo measurements. The described algorithms appear to be robust and lead to coherent results. Provisions are made in the method for the subsequent inclusion of a priori data to relieve the current requirements on the movements that can be studied with this technique.

\section{Acknowledgments}

We thank S. Lin for the transgenic $\operatorname{Tg}($ gata $1:$ GFP) zebrafish line. We thank Mike Tyszka for his comments on the manuscript. M. Liebling is supported by a fellowship from the Swiss National Science Foundation, PBEL2-104418.

\section{Appendix: Periodic Affine Transformations}

The transformation matrix in Eq. (28) can be decomposed using the following matrices for scaling

$$
\mathbf{C}(t)=\left(\begin{array}{cccc}
S_{x}(t) & 0 & 0 & 0 \\
0 & S_{y}(t) & 0 & 0 \\
0 & 0 & S_{z}(t) & 0 \\
0 & 0 & 0 & 1
\end{array}\right),
$$

translation

$$
\mathbf{T}(t)=\left(\begin{array}{cccc}
1 & 0 & 0 & T_{x}(t) \\
0 & 1 & 0 & T_{y}(t) \\
0 & 0 & 1 & T_{z}(t) \\
0 & 0 & 0 & 1
\end{array}\right),
$$

shear

$$
\mathbf{H}(t)=\left(\begin{array}{cccc}
1 & S_{x y}(t) & S_{x z}(t) & 0 \\
0 & 1 & S_{y z}(t) & 0 \\
0 & 0 & 1 & 0 \\
0 & 0 & 0 & 1
\end{array}\right),
$$

and rotation

$$
\begin{gathered}
\mathbf{R}(t)=\left(\begin{array}{cccc}
\cos [\psi(t)] & \sin [\psi(t)] & 0 & 0 \\
-\sin [\psi(t)] & \sin [\psi(t)] & 0 & 0 \\
0 & 0 & 1 & 0 \\
0 & 0 & 0 & 1
\end{array}\right) \\
\left(\begin{array}{cccc}
1 & 0 & 0 & 0 \\
0 & \cos [\vartheta(t)] & \sin [\vartheta(t)] & 0 \\
0 & -\sin [\vartheta(t)] & \sin [\vartheta(t)] & 0 \\
0 & 0 & 0 & 1
\end{array}\right) \\
\left(\begin{array}{cccc}
\cos [\phi(t)] & \sin [\phi(t)] & 0 & 0 \\
-\sin [\phi(t)] & \sin [\phi(t)] & 0 & 0 \\
0 & 0 & 1 & 0 \\
0 & 0 & 0 & 1
\end{array}\right),
\end{gathered}
$$

where the twelve coefficients

$$
\begin{aligned}
\theta(t)= & {\left[\theta_{1}(t), \ldots, \theta_{12}(t)\right] } \\
= & {[\phi(t), \vartheta(t), \psi(t),} \\
& S_{x}(t), S_{y}(t), S_{z}(t),
\end{aligned}
$$




$$
\begin{aligned}
& S_{x y}(t), S_{x z}(t), S_{y z}(t), \\
& \left.T_{x}(t), T_{y}(t), T_{z}(t)\right]
\end{aligned}
$$

are periodic functions that can conveniently be expressed by their Fourier series

$$
\begin{gathered}
\theta_{i}(t)=a_{0 i}+\sum_{k=1}^{\infty} a_{k i} \cos [2 \pi(k / T) t]+\sum_{k=1}^{\infty} b_{k i} \sin [2 \pi(k / T) t] \\
i=1, \ldots, 12
\end{gathered}
$$

\section{References}

1. M. Minsky, "Memoir on inventing the confocal scanning microscope," Scanning 10(4), 128-138 (1988).

2. Carl Zeiss AG, "LSM 5 LIVE," http://www.zeiss.de/.

3. J. R. Hove, R. W. Köster, A. S. Forouhar, G. Acevedo-Bolton, S. E. Fraser, and M. Gharib, "Intracardiac fluid forces are an essential epigenetic factor for embryonic cardiogenesis," Nature (London) $\mathbf{4 2 1}$, 172-177 (2003).

4. E. A. V. Jones, M. H. Baron, S. E. Fraser, and M. E. Dickinson, "Measuring hemodynamic changes during mammalian development," Am. J. Physiol. Heart Circ. Physiol. 287(4), H1561-H1569 (2004).

5. R. Jerecic, M. Bock, S. Nielles-Vallespin, C. Wacker, W. Bauer, and L. R. Schad, "ECG-gated Na-23-MRI of the human heart using a 3D-radial projection technique with ultra-short echo times," Magn. Reson. Mater. Phys., Biol., Med. 16(6), 297-302 (2004).

6. M. Markl, F. P. Chan, M. T. Alley, K. L. Wedding, M. T. Draney, C. J. Elkins, D. W. Parker, R. Wicker, C. A. Taylor, R. J. Herfkens, and N. J. Pelc, "Time-resolved three-dimensional phase-contrast MRI," J. Magn. Reson Imaging 17(4), 499-506 (2003).

7. S. Skare and J. L. R. Andersson, "On the effects of gating in diffusion imaging of the brain using single shot EPI," Magn. Reson. Imaging 19(8), 1125-1128 (2001).

8. C. Dornier, M. K. Ivancevic, P. Thévenaz, and J. P. Vallée, "Improvement in the quantification of myocardial perfusion using an automatic spline-based registration algorithm," J. Magn. Reson Imaging 18(2), 160-168 (2003).

9. Z. P. Liang, H. Jiang, C. P. Hess, and P. C. Lauterbur, "Dynamic imaging by model estimation," Int. J. Imaging Syst. Technol. 8(6), 551-557 (1997).

10. T. Pan, T. Y. Lee, E. Rietzel, and G. T. Y. Chen, "4D-CT imaging of a volume influenced by respiratory motion on multi-slice CT," Med. Phys. 31(2), 333-340 (2004).

11. C. Le Rest, O. Couturier, A. Turzo, and Y. Bizais, "Postsynchronization of dynamic images of periodically moving organs," Nucl. Med. Commun. 21(7), 677-684 (2000).

12. R. B. Thompson and E. R. McVeigh, "Flow-gated phase-contrast MRI using radial acquisitions," Magn. Reson. Med. 52(3), 598-604 (2004).

13. M. E. Crowe, A. C. Larson, Q. Zhang, J. Carr, R. D. White, D. B. Li, and O. P. Simonetti, "Automated rectilinear self-gated cardiac cine imaging," Magn. Reson. Med. 52(4), 782-788 (2004).

14. A. C. Larson, R. D. White, G. Laub, E. R. McVeigh, D. B. Li, and O. P. Simonetti, "Self-gated cardiac cine MRI," Magn. Reson. Med. 51(1), 93-102 (2004).

15. T. A. Spraggins, "Wireless retrospective gating-application to cine cardiac imaging," Magn. Reson. Imaging 8(6), 675-681 (1990).

16. R. D. White, C. B. Paschal, M. E. Clampitt, T. A. Spraggins, and G. W. Lenz, "Electrocardiograph-independent, wireless cardiovascular cine MR imaging," J. Magn. Reson Imaging 1(3), 347-355 (1991).

17. M. Grass, R. Manzke, T. Nielsen, P. Koken, R. Proksa, M. Natanzon, and G. Shechter, "Helical cardiac cone beam reconstruction using retrospective ECG gating," Phys. Med. Biol. 48(18), 3069-3084 (2003).

18. M. Kachelrieß, D. A. Sennst, W. Maxlmoser, and W. A. Kalender, "Kymogram detection and kymogram-correlated image reconstruction from subsecond spiral computed tomography scans of the heart," Med. Phys. 29(7), 1489-1503 (2002).

19. S. A. de Winter, R. Hamers, M. Degertekin, K. Tanabe, P. A. Lemos, P. W. Serruys, J. R. T. C. Roelandt, and N. Bruining, "Retrospective image-based gating of intracoronary ultrasound images for improved quantitative analysis: the intelligate method," Catheterization Cardiovascular Interventions 61(1), 84-94 (2004).

20. G. M. Treece, R. W. Prager, A. H. Gee, C. J. C. Cash, and L. Berman, "Grey-scale gating for freehand 3D ultrasound," in Proc. First 2002 IEEE Int. Symp. Biomed. Imag.: Marco to Nano, pp. 993-996, Washington DC, USA (2002).

21. Q. Tian and M. N. Huhns, "Algorithms for subpixel registration," Comput. Vis. Graph. Image Process. 35(2), 220-233 (1986).

22. L. G. Brown, "A survey of image registration techniques," ACM Comput. Surv. 24(4), 325-376 (1992).

23. C. Stiller and J. Konrad, "Estimating motion in image sequences-a tutorial on modeling and computation of 2D motion," IEEE Signal Process. Mag. 16(4), 70-91 (1999).

24. J. L. Barron, D. J. Fleet, and S. S. Beauchemin, "Performance of optical-flow techniques," Int. J. Comput. Vis. 12(1), 43-77 (1994).

25. J. Cox and C. J. R. Sheppard, "Digital image processing of confocal images," Image Vis. Comput. 1(1), 52-56 (1983).

26. A. Diaspro, "Introduction: image processing in optical microscopy," Microsc. Res. Tech. 64(2), 87-88 (2004).

27. Y. L. Sun, B. Rajwa, and J. P. Robinson, "Adaptive image-processing technique and effective visualization of confocal microscopy images," Microsc. Res. Tech. 64(2), 156-163 (2004).

28. M. Kozubek, P. Matula, P. Matula, and S. Kozubek, "Automated acquisition and processing of multidimensional image data in confocal in vivo microscopy," Microsc. Res. Tech. 64(2), 164-175 (2004).

29. R. L. Allen, F. A. Kamangar, and E. M. Stokely, "Laplacian and orthogonal wavelet pyramid decompositions in coarse-to-fine registration," IEEE Trans. Signal Process. 41(12), 3536-3541 (1993).

30. M. J. Carroll and K. E. Britton, "Wavelet based image registration and its applications in nuclear medicine," Eur. J. Nucl. Med. 26(9), 1008-1008 (1999).

31. J. Deubler and J. C. Olivo, "A wavelet-based multiresolution method to automatically register images," J. Math. Imaging Vision 7(3), 199_ 209 (1997).

32. I. D. Dinov, M. S. Mega, T. Poon, P. Thompson, and A. W. Toga, "Volumetric registration of brain data: a new technique for unbiased assessment and comparison of different registration methods using efficient wavelet representation," Neurology 58(7), A141-A141 (2002).

33. S. Gefen, O. Tretiak, L. Bertrand, G. D. Rosen, and J. Nissanov, "Surface alignment of an elastic body using a multiresolution wavelet representation," IEEE Trans. Biomed. Eng. 51(7), 1230-1241 (2004).

34. J. Le Moigne, W. J. Campbell, and R. F. Cromp, "An automated parallel image registration technique based on the correlation of wavelet features," IEEE Trans. Geosci. Remote Sens. GE-40(8), 1849-1864 (2002)

35. R. Sharman, J. M. Tyler, and O. S. Pianykh, "A fast and accurate method to register medical images using wavelet modulus maxima," Pattern Recogn. Lett. 21(6-7), 447-462 (2000).

36. M. Unser, P. Thévenaz, C. H. Lee, and U. E. Ruttimann, "Registration and statistical-analysis of PET images using the wavelet transform," IEEE Eng. Med. Biol. Mag. 14(5), 603-611 (1995).

37. M. M. Dworetsky, "A period-finding method for sparse randomly spaced observations or how long is a piece of string," Mon. Not. R. Astron. Soc. 203(3), 917-924 (1983).

38. R. F. Stellingwerf, "Period determination using phase dispersion minimization," Astrophys. J. 224(3), 953-960 (1978).

39. Matlab Function Reference, Vol. 2: F-O, The MathWorks, Inc., Natick, MA (2002).

40. R. P. Brent, Algorithms for Minimization without Derivatives, Prentice Hall, Englewood Cliffs, NJ (1973).

41. W. H. Press, S. A. Teukolsky, W. T. Vetterling, and B. P. Flannery, Numerical Recipes in C, 2nd ed., Cambridge University Press, Cambridge (1992).

42. E. H. W. Meijering, W. J. Niessen, and M. A. Viergever, "Quantitative evaluation of convolution-based methods for medical image interpolation," Med. Image Anal 5(2), 111-126 (2001).

43. P. Thévenaz, T. Blu, and M. Unser, "Image interpolation and resampling," in Handbook of Medical Imaging, Processing and Analysis, I. Bankman, Ed., Chap. 25, pp. 393-420, Academic Press, San Diego, CA (2000).

44. P. Thévenaz, U. E. Ruttimann, and M. Unser, "A pyramid approach to subpixel registration based on intensity," IEEE Trans. Image Process. 7(1), 27-41 (1998). 
45. S. G. Mallat, "A theory for multiresolution signal decompositionthe wavelet representation," IEEE Trans. Pattern Anal. Mach. Intell. 11(7), 674-693 (1989).

46. D. L. Donoho and I. M. Johnstone, "Ideal spatial adaptation by wavelet shrinkage," Biometrika 81(3), 425-455 (1994).

47. J. B. Weaver, X. Yansun, D. M. Healy, Jr., and L. D. Cromwell, "Filtering noise from images with wavelet transforms," Magn. Reson. Med. 21(2), 288-295 (1991).

48. M. Antonini, M. Barlaud, M. Mathieu, and I. Daubechies, "Image coding using wavelet transform," IEEE Trans. Image Process. 1(2), 205-220 (1992).

49. M. Unser and T. Blu, "Mathematical properties of the JPEG2000 wavelet filters," IEEE Trans. Image Process. 12(9), 1080-1090 (2003).

50. C. M. Brislawn, "Classification of nonexpansive symmetric extension transforms for multirate filter banks," Appl. Comput. Harmon. Anal. 3(4), 337-357 (1996).

51. Bitplane AG, "Imaris," http://www.bitplane.ch/.

52. S. R. Sternberg, "Biomedical image-processing," Computer 16(1), 22-34 (1983).

53. W. Rasband, "ImageJ," http://rsb.info.nih.gov/ij/.

54. E. D. Angelini, A. F. Laine, S. Takuma, J. W. Holmes, and S. Homma, "LV volume quantification via spatiotemporal analysis of real-time 3-D echocardiography," IEEE Trans. Med. Imaging 20(6),
457-469 (2001).

55. R. M. Willett and R. D. Nowak, "Platelets: a multiscale approach for recovering edges and surfaces in photon-limited medical imaging," IEEE Trans. Med. Imaging 22(3), 332-350 (2003).

56. M. Unser, "Splines: a perfect fit for signal and image processing," IEEE Signal Process. Mag. 16(6), 22-38 (1999).

57. M. Sermesant, C. Forest, X. Pennec, H. Delingette, and N. Ayache, "Deformable biomechanical models: application to 4D cardiac image analysis," Med. Image Anal 7(4), 475-488 (2003).

58. J. D. Foley and A. Van Dam, Fundamentals of Interactive Computer Graphics, Addison-Wesley, Reading, MA (1984).

59. D. Robinson and P. Milanfar, "Fundamental performance limits in image registration," IEEE Trans. Image Process. 13(9), 1185-1199 (2004).

60. Q. M. Long, A. M. Meng, H. Wang, J. R. Jessen, M. J. Farrell, and S. Lin, "GATA-1 expression pattern can be recapitulated in living transgenic zebrafish using GFP reporter gene," Development 124(20), 4105-4111 (1997).

61. A. S. Forouhar, M. Liebling, R. Wolleschensky, R. Ankerhold, B. Zimmermann, S. E. Fraser, M. Gharib, and M. E. Dickinson, "Rapid, confocal microscopy reveals functional changes in embryonic heart development," (in preparation).

62. http://bioimaging.caltech.edu/publications/jbo2005/ 